\title{
Low power real-time data acquisition using compressive sensing
}

Linda S. Powers, Yiming Zhang, Kemeng Chen, Huiqing Pan, Wo-Tak Wu, et al. 


\title{
Low Power Real-time Data Acquisition using Compressive Sensing
}

\author{
Linda S. Powers ${ }^{\mathrm{abc}}$, Yiming Zhang ${ }^{\mathrm{a}}$, Kemeng Chen ${ }^{\mathrm{a}}$, Huiqing Pan ${ }^{\mathrm{a}}$, Wo-Tak Wu ${ }^{\mathrm{a}}$, Peter W. Hall ${ }^{\mathrm{a}}$, \\ Jerrie V. Fairbanks ${ }^{\mathrm{a}}$, Radik Nasibulin ${ }^{\mathrm{a}}$ and Janet M. Roveda ${ }^{\text {abc }}$ \\ ${ }^{a}$ Dept. of Electrical and Computer Engineering, \\ ${ }^{\mathrm{b}}$ Biomedical Engineering Graduate Interdisciplinary Program, ${ }^{\mathrm{c}}$ Bio5 Institute \\ The University of Arizona, Tucson, AZ 85721
}

\begin{abstract}
New possibilities exist for the development of novel hardware/software platforms having fast data acquisition capability with low power requirements. One application is a high speed Adaptive Design for Information (ADI) system that combines the advantages of feature-based data compression, low power nanometer CMOS technology, and stream computing [1]. We have developed a compressive sensing (CS) algorithm which linearly reduces the data at the analog front end, an approach which uses analog designs and computations instead of smaller feature size transistors for higher speed and lower power. A level-crossing sampling approach replaces Nyquist sampling. With an in-memory design, the new compressive sensing based instrumentation performs digitization only when there is enough variation in the input and when the random selection matrix chooses this input.
\end{abstract}

Keywords: Compressive sensing, Adaptive Design for Information, low power, real-time data acquisition

\section{INTRODUCTION}

New possibilities exist for the development of novel hardware/software platforms having fast data acquisition capability with low power requirements. One application is a high speed Adaptive Design for Infor mation (ADI) system that combines the advantages of feature-based data compression, low power nanometer CMOS technology, and stream computing [1]. Typical data acquisition systems use a sensor together with a mixed signal op-amp and ADC feed to a stream processor. In this approach, the front end analog portion consumes more than half the total chip area and is several orders of magnitude slower than the digital portion of the system.

We have developed a compressive sensing (CS) algorithm which linearly reduces the data at the analog front end, an approach which uses analog designs and computations instead of smaller feature size transistors for higher speed and lower power. A level-crossing sampling approach replaces Nyquist sampling. With an in-memory design, the new compressive sensing based instrumentation performs digitization only when there is enough variation in the input and when the random selection matrix chooses this input. For example, fluorescence data can not only be collected in realtime, but also analyzed in real-time with these methods to provide lifetimes of specific fluorophores. While CS is a means by which $\mathrm{N}$-dimensional data can be transformed by a linear operator into data in an M-dimensional vector space, where $\mathrm{M} \ll \mathrm{N}$, the M-dimensional data in common CS practice does not have any semantic meaning. Thus, characterization of a point in a random matrix usually relies on machine learning algorithms to cluster data points. In this paper, we discuss how to incorporate meaning into the resulting M-tuples obtained when applying the linear operator to the original uncompressed $\mathrm{N}$-tuples, so that information contained in the original data can be extracted from the compressed data without utilizing a clustering algorithm. One type of data considered here are exponentially decaying waveforms, and the linear operator employed is $T$ (time-constant for the decaying exponential function) which is extracted by transforming the original $\mathrm{N}$-dimensional data into a subspace of a random matrix where each element of the M-tuple that resides in this subspace is a close approximation to the decay time constant $T$. Another approach utilizes mono-exponential decay estimates from a rapid lifetime determination algorithm [2] to extract $T$ from the compressed M-dimensional data. However, multiple component exponential data have not been analyzed in this manner.

*email: 1spowers @email.arizona.edu; phone: (520) 621-7634 ; website: http://ece.arizona.edu/linda-s-powers

Micro- and Nanotechnology Sensors, Systems, and Applications IX, edited by Thomas George,

Achyut K. Dutta, M. Saif Islam, Proc. of SPIE Vol. 10194, 101940C · @ 2017 SPIE

CCC code: $0277-786 \mathrm{X} / 17 / \$ 18 \cdot$ doi: $10.1117 / 12.2263220$

Proc. of SPIE Vol. 10194 101940C-1 


\section{MODEL BASED COMPRESSIVE SENSING FOR EXPONENTIAL DECAY}

\subsection{What is compressive sensing?}

Let us begin with a brief review of compressive sensing theory [2,4-7]. An example image can be presented as an NxM matrix. By using a random selection matrix, we can generate a new matrix :

$$
y_{m x M}=[\Phi]_{m x N}[X]_{N x M}(1) \text {. }
$$

Because $m<<N$, we reduce the total data amount. Different from JPEG and other nonlinear co mpression algorith ms, compressive sensing linearly reduces data and preserves key features without much distortion. This is the key reason why compressive sensing can be applied to the front end of a data acquisition system instead of right before data transmission. One such example is a "single pixel camera" [9]. The camera performs random selection on the sampled object. Thus, a smaller amount of data will be generated by the camera and subsequently enter the following data acquisition system. Depending on the sparsity of the sampled data, the average data reduction by compressive sensing is about 50\%. If we use joule per bit as an energy estimation, this indicates that this compression algorithm may lead to a total energy/power reduction for the following data processing architecture.

In this paper, we extend the compressive sensing algorithm to real applications. Model based compressive sensing algorith ms are used to leverage the structure present in signals obtained by the sensors. In particular, several common real application signals such as exponential decaying signals and periodic physiological signals (i.e. ECG waveforms) are investigated.

\subsection{Model based compressive sensing}

The traditional compressive sensing theorem assumes that coefficients of some transforms performed on measure d data (e.g., wavelet, DCT) are K-sparse, where $K$ is much smaller than the data size $N$ [2]. Thus, the data can be represented using only $K$ samples. Model based compressive sensing proposed in [3] explored the structure of data to be sampled and further reduced $\mathrm{K}$ without sacrificing the recovery performance. The key idea is to relax the restricted isometric property (RIP) [4] of the random sampling matrix and employ a structure based recovery algorithm according to a data model. In the current applications, the signals collected are an exponential decay waveform and a periodic waveform, both mixed with noise. Our sampling strategy enables a high compression rate via RIP relaxation and achieves denoising recovery to some extent by enhancing coefficient sparsity in recovery. We first review the RIP presentation and then show the details of our sampling strategy. In traditional compressive sensing, the sampling matrix follows the RIP such that original information could be recovered. Mathematically, the RIP is represented as equation (2)

$$
\left(1-\delta_{k}\right)\|x\|_{2}^{2} \leq\|\Phi x\|_{2}^{2} \leq\left(1+\delta_{k}\right)\|x\|_{2}^{2}
$$

where $\boldsymbol{\Phi}$ is the random sampling matrix of size $M$ by $N, x$ is the data size $N$, and $\delta_{\mathrm{k}}$ is a constant. Under the RIP constraint, the random matrix ensures the relative distance within $x$ which will still be preserved after sampled by $\boldsymbol{\Phi}$. Thus, information within $\mathrm{x}$ is also preserved after the sampling procedure $\boldsymbol{\Phi}$. To enable higher compression rates, we relax the RIP. This gives more flexibility to the sampling matrix $\boldsymbol{\Phi}$. The general RIP requires sparsity of data $x$ before compression. However, when the data information is modeled as $x_{\text {model }}$, we can further relax $\delta_{\mathrm{k}}$ in (1) which provides a lower sampling rate than that of the orig inal RIP. The recovery process generates the sparsest solution which also satisfies the compression equation.

Nevertheless, in a real data acquisition system, the data from circuits is always mixed with noise. Thus, we must develop a noise removal recovery model for our data acquisition system. Equations (3) and (4) define the recovery model using a model based sampling matrix, $\boldsymbol{\Phi}_{\mathrm{m}}$ and an orthogonalbasis, $\Psi$, therefore transforming $x$ into coefficients $\alpha$.

$$
\begin{gathered}
y=\Phi_{m} \Psi \alpha \text { where } x=\Psi \alpha(3) \\
\hat{\alpha}=\min \|\alpha\| \text { s.t. }\left\|y-\Phi_{m} \Psi \hat{\alpha}\right\| \leq \varepsilon(4)
\end{gathered}
$$

As denoted in (3), $x$ is the data collected directly from the noisy instrument. The coefficient $\alpha$ also contains noise. Next, we recover to a noise-free solution, $\hat{x}$, whose coefficients under the $\Psi$ transform is $\hat{\alpha}$. This is described in (4). $\hat{\alpha}$ is the noise free coefficient and $\varepsilon$ is the error tolerance. In the recovery procedure, our aim is to look for the sparsest coefficients. These coefficients must lie within the recovery error tolerant value $\varepsilon$ determined by the instrument signal- 
to-noise ratio. The recovery procedure is established based on the assumption that the sparsity of data is much higher than noise. Therefore, given a tolerance value correspond ing to the instrument noise level, the recovered coefficient will be sparserthan without a tolerance, which is more likely to represent the true information.

\section{DISCUSSION \& CONCLUSION}

Two different applications are shown to illustrate the concept of model-based compressive sensing: an exponential decay from a time-resolved fluorescence measurement and an electrocard iogram (ECG) signal. In the first application, the signal collected is an exponential decay waveform mixed with noise. As is well known, wide spectrum signals can appear in ultra-broadband communications, mo lecular fluorescent decay signatures, imag ing and vision systems for high speed aircrafts, and other physical phenomena, some of which have not been able to be measured directly because of the required sampling speed. Of particular interest to us are applications of this methodology to biomedical problems. Hence, an exponentially decaying signal and a repetitive signal are chosen to demonstrate the model-based compressive sensing strategy. In the exponential decay, the signal acquired is approximated by an exponential decay with instrumental noise. Since information contained in the data is represented by the corresponding entries of the sampling matrix values, there is a high probability that higher weights are on the part of the data which contains more information. Thus, the compressed data mainly contains critical information which is sparser than noise. For these reasons, the recovery process is more likely to recover only the most important information.

Figure 1 shows the recovery result using noisy experimental data from a time-resolved fluorescence instrument. Since the model based sampling matrix places less weight on noise, the noise has only a little impact on the result. The blue solid line is the data collected directly from the instrument and red dashed line represents the recovered data.

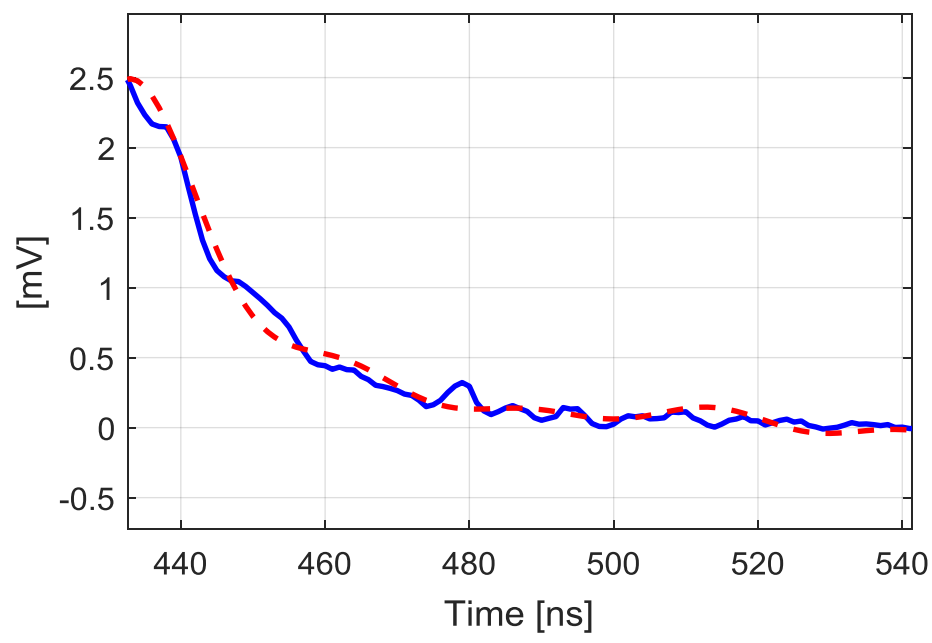

Figure 1. Comparison of the raw data (blue solid line) and recovery data (red dash line) showing the de-noising performance.

The time resolved fluorescence measurement is ethidium bromide bound to DNA in $\mathrm{D}_{2} \mathrm{O}$. For this application, the data is a single exponential measured by a time-resolved fluorometer and the input data consists of 143 points. In the first step, the DCT basis is applied to the input signal $\mathrm{x}$, transforming $\mathrm{x}$ into the coefficient vector $\alpha$ which has a sparsity greater than x. A Gaussian random matrix is selected. Based on the sparsity of the coefficient vector $\alpha$, we can choose the amount of compression for the signal. In this application, the data is compressed from 143 points to 15 points. After the compression step, the CoSaMP algorithm is applied to the compressed data to reconstruct the original data. Figure 1 shows the original input data and the reconstructed data and Figure 2 shows the difference. After this, both data, the curve fitting for orig inal input data and reconstructed data, are fitted to an exponential function to determine the decay constant $T$. For this application, the decay constants are approximately equal to $6.9 \mathrm{~ns}$ for both original input data and reconstructed data. This value is in good agreement with that reported in the literature [10]. 


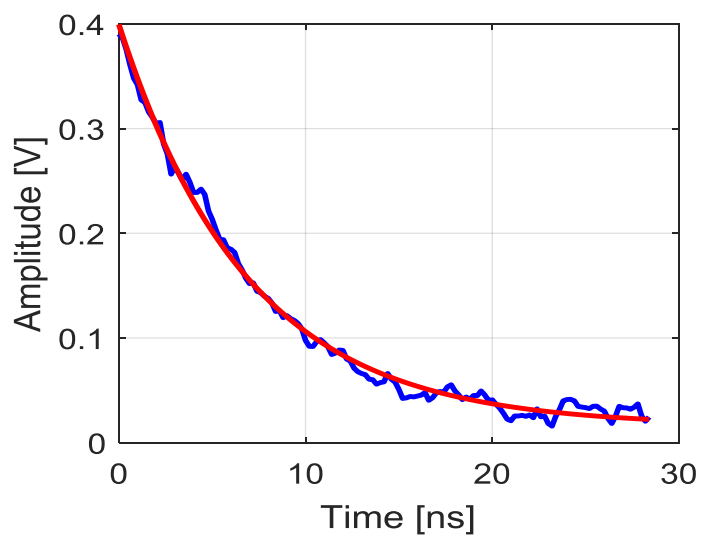

(a)

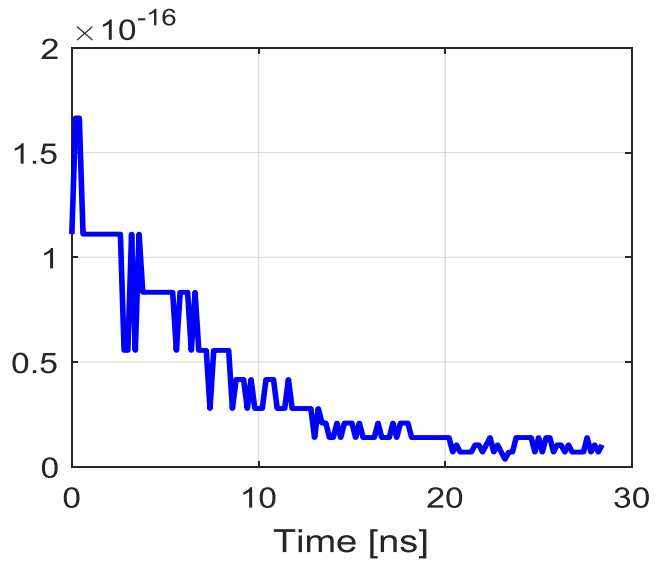

(b)

Figure 2. Reconstructed data (red) from the compressive sensing exponential decay algorithm compared to the original data (blue) (a) and the difference between the original exponential decay data and the reconstructed data (b).

For the ECG application, the data is a periodic waveform with QRST features measured by an ECG circuit. The input data consists of 512 samples, about three periods from a set of 249250 raw data samples with $256 \mathrm{sps}$. In the first step, the wavelet basis is applied to the input data, $\mathrm{x}$, transforming $\mathrm{x}$ into coeffic ient vector $\alpha$ which has a sparsity greater than $x$. Note that this wavelet basis is created based on Daubechies wavelet function [11] due to its regularity which aids in the rentention of the smoothness of the original data. A Gaussian random matrix is then applied as our selection matrix because it is incoherent with the Daubechies wavelet basis. In addition, this basis also preserves the spars ity before and after projection onto this basis. Based on the sparsity of the coefficient vector $\alpha$, we can choose the amount of compression for the signal. In this application, the signal is compressed from 512 samples to 256 samples. After the compression step, the CoSaMP algorithm is applied to the compressed signal to reconstruct the original signal. The relative error is $R_{e r}=\frac{\operatorname{norm}(\hat{x}-x)}{\operatorname{norm}(x)}=0.0013$.

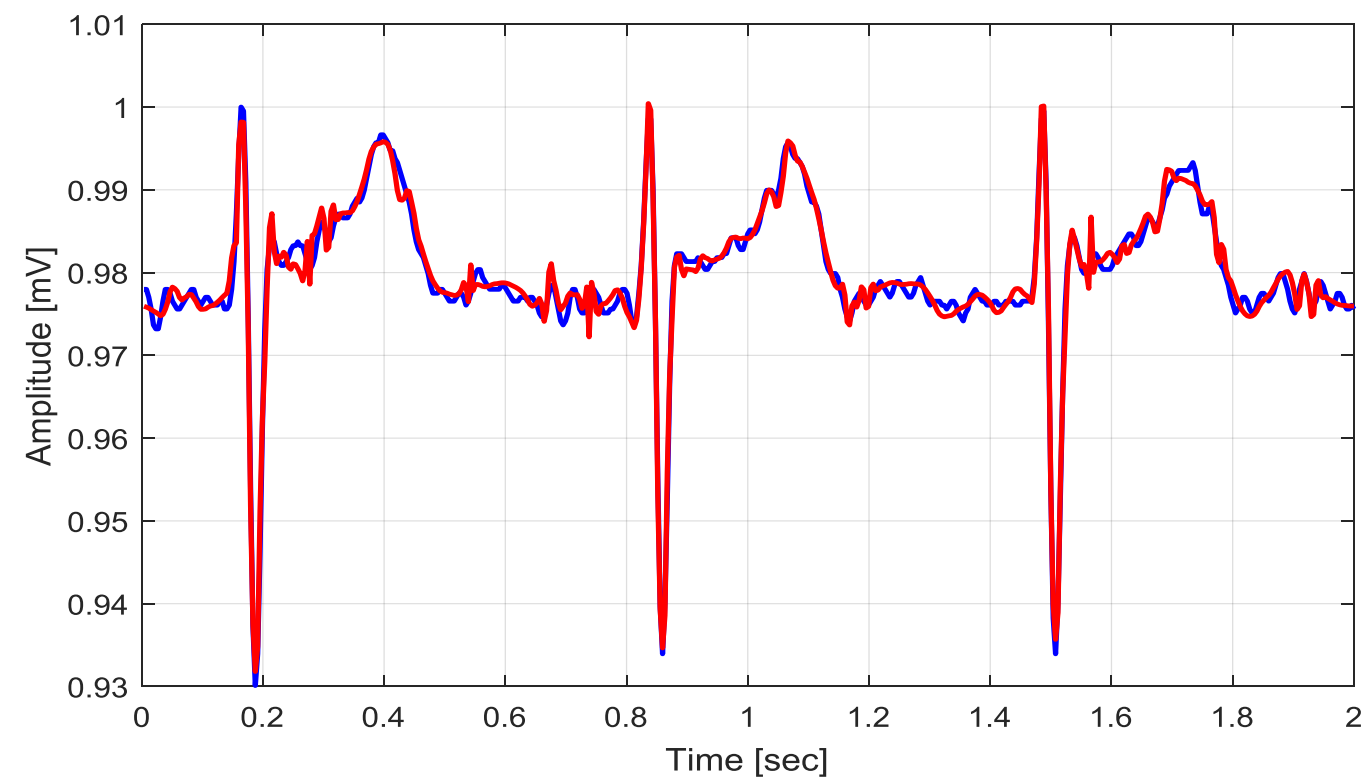

Figure 3. Wavelet model based compressive sensing of ECG data. The blue line represents the original data. The red line represents the recovered data using CoSaMP. 


\section{OUTLOOK}

The massive data explosion in both quantity and diversity of mobile, wireless and cloud based devices is the direct consequence of affordable, ubiquitous digital computing due to innovations in integrated circuit (IC) technologies. Previous generations generated only a small amount of analog data, but now we are facing an enormous volume of digital data produced from diverse sources through a variety of channels, preventing real-time analysis using traditional data acquisition architectures and designs. A major concern raised by many is whether we can actually get any useful information from such large pools of data. Data mining, a methodology that uses models to extract information from data, can be effective in presenting a summary of the data or the most extreme features of the data, depending on the appropriate selection of models $[12,14,16,17]$. Searching for certain patterns or occurrences in data also faces challenges. According to Bonferroni's theory $[12,16,17]$, when the data volume increases, the number of certain occurrences also grows. A mong highly diversified data, the occurrences become statistical artifacts rather than evidence of the searching patterns. The only way to avoid data explosion is to perform streamline computing for cognitive data acquisition from the very beginning [12-18]. Through model based compressive sensing, this paper demonstrated how to cognitively reduce the data volume at the sensor and system level while maintaining the data structure and accuracy. Our preliminary results show that it is possible to provide structured data with much lower data volume. We believe that our investigations provide a starting point for real-time data acquisition in large data volu me applications such as mobile health, telemedicine, and precision medicine.

\section{ACKNOWLEDGEMENT}

The authors thank Dr. Walther R. Ellis, Jr. for his support.

\section{REFERENCES}

[1] Ko, J., Lu, C., Srivastava, M., Stankovic, J., Terzis, A., and Welsh, M., "Wire less Sensor Networks for Healthcare", Proceedings of the IEEE, Vol. 98, No. 11, Nov. 2010.

[2] D. L. Donoho, “Compressed sensing," IEEE Trans. Inf. Theory, vol. 52, no. 4, pp. 1289-1306, Sep. 2006.

[3] Richard G. Baraniuk, Volkan Cevher, Marco F. Duarte, Chin may Hegde, "Model-Based Compressive Sensing", IEEE Transactions on Information Theory, vol. 56, No. 4, April 2010.

[4] E. J. Candès, "Compressive sampling," in Proc. Int. Congr. Math., Madrid, Spain, 2006, vol. 3, pp. 1433-1452.

[5] J. A. Tropp, J. N. Laska, M. F. Duarte, J. K. Romberg, and R. G. Baraniuk. Beyond Nyquist: Efficient sampling of sparse bandlimited signals. IEEE Trans. Inform. Theory, 2010, 56(1): 520-544.

[6] Richard Baraniuk, Mark Davenport, Ronald De Vore, and Michael Wakin. A simple proof of the restricted isometry property for random matrices. Constructive Approximation, 2008, 28(3): 253-263.

[7] D. Needell, J.A. Tropp. CoSaMP: Iterative signal recovery from incomplete and inaccurate samples. Applied and Computational Harmonic Analysis, 2009,26(3):301-321

[8] Akcakaya M., Seunghoon Nam, Peng Hu, Moghari M. H., Ngo L.H., Tarokh V., Manning W.J., Nezafat R.. Compressed sensing with wavelet do main dependencies for coronary MRI: A retrospective study. Medical Imaging, IEEE Transactions on, 2011, 30(5): 1090-1099.

[9] http://dsp.rice.edu/cscamera

[10] John Olmsted III and David R. Kearns, Mechanism of Eth idium Bromide Fluorescence Enhancement on Binding to Nucleic Acids, Biochemistry 16 (16), 1977, 3647.

[11] Daubechies, Ten Lectures on Wavelets, SIAM, 1992, p. 194.

[12] Mining of Massive Datasets, Anand Rjaraman, Jure Leskoves, Jeffrey D. Ullman, Stanford Univ. 2012.

[13] A. Broder, R. Kumar, F. Maghoul, P. Raghavan, S. Rajagopalan, R. Stata, A. Tomkins, and J. Weiner, "Graph structure in the web," Computer Networks 33:1-6, pp. 309-320, 2000.

[14] M.M. Gaber, Scientific Data Mining and Knowledge Discovery — Principles and Foundations, Springer, New York, 2010.

[15] H. Garcia-Molina, J.D. Ullman, and J. Widom, Database Systems: The Complete Book Second Edition, PrenticeHall, Upper Saddle River, NJ,2009.

[16] C.P. Manning, P. Raghavan, and H. Schüutze, Introduction to Information Retrieval, Cambridge Univ. Press, 2008. 
[17] P.-N. Tan, M. Steinbach, and V. Kumar, Introduction to Data Mining, Addison-Wesley, Upper Saddle River, NJ, 2005.

[18] Stream Computing: Real-time Processing of Massive Data, NEC Annual R\&D Meeting, 2009.

Proc. of SPIE Vol. 10194 101940C-6 